\title{
JOINING THE RHIC ONLINE AND OFF-LINE MODELS*
}

\author{
N.Malitsky", K.Brown, N.D’Imperio, J. Kewisch, A.Fedotov, A.Luccio, F.Pilat, V.Ptitsyn, \\ T.Satogata, S.Tepikian, J.Wei, BNL, Upton, NY 11973 \\ R. Talman, Cornell University, Ithaca, NY 14853
}

\begin{abstract}
The paper presents an interface encompassing the RHIC online ramp model and the UAL off-line simulation framework. The resulting consolidating facility aims to minimize the gap between design and operation data as well as to facilitate analysis of the RHIC performance and future upgrades. The interface is based on the Accelerator Description Exchange Format (ADXF[1]) that represents a snapshot of the RHIC online model. The same approach is also considered for integrating the AGS and RHIC modeling environments.
\end{abstract}

\section{MOTIVATION}

Over the past several years, the RHIC online model has been continuously developed and employed in resolving various commissioning and operation problems. Up till now, the online model has successfully reproduced linear optics within needed accuracy, which is critical for tune, orbit, and chromaticity corrections. However, a consistent effort towards higher luminosity and future RHIC II and eRHIC projects bring new challenges to the modeling applications. Advanced beam dynamics studies require significant resources and are usually conducted off-line. On the other hand, the accuracy of accelerator simulation results is very sensitive to a correct machine description used in accelerator operations. Online and off-line models complement each other and their direct connection is advantageous in analysis of complex beam dynamics problems. Originally, the RHIC models were initialized from a design database through different chains of data transformations. A task has now been implemented with the common Accelerator Description Exchange Format (ADXF[1]) file which accommodates a snapshot of the RHIC operational lattice. In the following sections different parts of the RHIC joined modeling environment are described. We conclude with the early results and future plans.

\section{ONLINE MODEL FACILITY}

The RHIC online control facility is built after the standard three-tier architecture, encompassing Wave Form Generators (WFG) controlling power supplies, several middle-layer servers, and an open collection of client applications (see Fig. 1). Communication among distributed components is implemented with the CDEV message-oriented middleware [2]. The Ramp Manager is a central server in this infrastructure. It encapsulates ramp settings, and provides all computational models and user applications with a consistent set of magnet strength values. Accelerator optics parameters (such as tunes, chromaticities, Twiss functions, transfer matrices, etc.) are calculated by the dedicated Online Model Server hosting the Accelerator Computational Model Engine (ACME [3]). The RHIC online framework supports multiple servers, each implementing a common ACME interface. Such flexible open infrastructure facilitates debugging of modeling algorithms and selecting the most optimal engines for particular applications. The client applications are written using the CDEV Application Programming Interface (API) which provides unified access to online primary and/or calculated data. Several dozens of client applications have been implemented and successfully employed during the RHIC commissioning and operations, including Ramp Editor, Injection Application, Orbit Correction, Transverse Profile Manager, and many others.

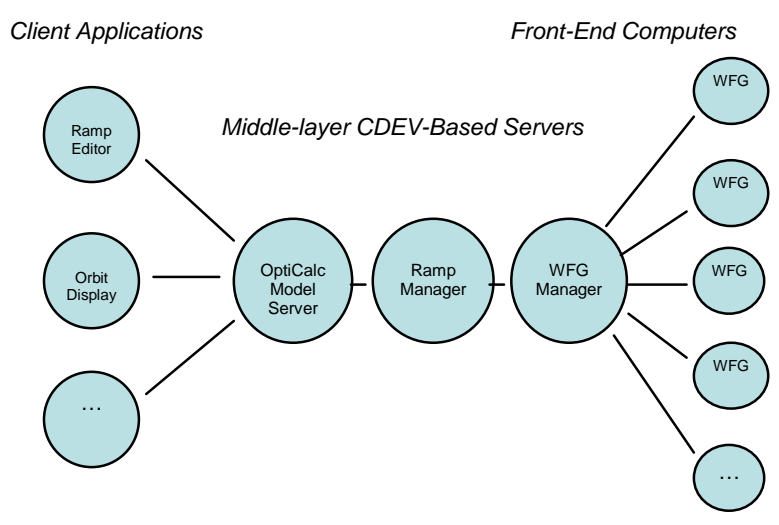

Figure 1: RHIC three-tier online model facility.

In RHIC, a ramp model [4] is represented by a sequence of stepping stones, each containing a set of magnet strengths at a point in time. Magnet settings between such points are calculated from an appropriate interpolation. The stepping stone content corresponds to the traditional lattice description used in accelerator design and simulation programs. Then all optics calculations, tuning, comparison of model results with instrumentation measurements are associated with a particular stepping stone. For regular machine operation, the fastest computational accelerator algorithms are preferred and the RHIC online model server employs an efficient matrix-based OptiCalc engine dealing with linear optics and sextupole effects. Because the OptiCalc data corresponds to the content of a single stepping stone, the online side of the ADXF-based interface has been

\footnotetext{
*Work performed under the auspices of the U.S. Department of Energy \#malitsky@bnl.gov
} 
narrowed to the OptiCalc program and implemented as a writer of its corresponding containers.

\section{UAL SIMULATION ENVIRONMENT}

The Unified Accelerator Libraries (UAL[5]) environment addresses the complex simulation tasks of modern beam dynamics studies. It offers an open collection of accelerator algorithms and a consistent mechanism for building configurable project-specific accelerator off-line models. A cornerstone of this mechanism is the Element-Algorithm-Probe framework [6] which identifies the association among three major concepts. The Element part represents accelerator magnets and devices. In UAL, a hierarchical tree of accelerator components is organized as the Standard Machine Format (SMF) module. All accelerator propagators are derived from a basis class Algorithm and are initially separated from the accelerator elements. In applications, algorithms can be dynamically loaded and connected in the accordance with the user text file written in Accelerator Propagator Description Format (APDF). Probes could be any objects (e.g. Bunch, Twiss function, Taylor maps, etc.) evolved by the corresponding algorithms.

Recently, UAL has been integrated with the QT GUI development framework and the ROOT analysis environment [7]. The new package extends the UAL simulation algorithms with an open collection of analysis and visualization components. The original Perl-based scripting interface has also been transformed into a GUI application. Its main window is implemented as a configurable and interactive Accelerator Physics Player (see Fig 2.) which coordinates data flows among tracking engines, interactive graphics, and data-processing.

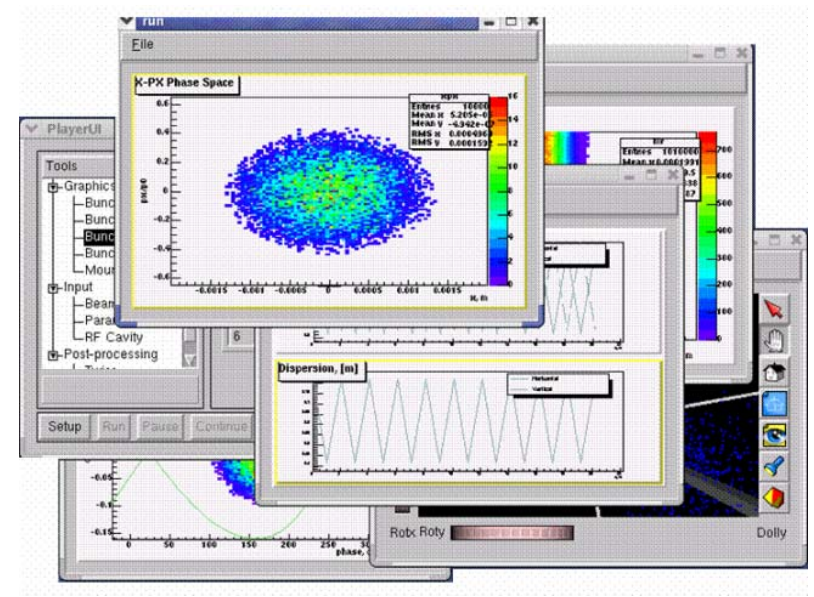

Figure 2: Interactive physics player with a set of viewers.

Because of a wide spectrum of supporting applications, UAL is more complex then the OptiCalc online model. But the task associated with the ADXF interface is very similar and is encapsulated in the implementation of the ADXF-based reader for the SMF accelerator containers.

\section{ACCELERATOR EXCHANGE FORMAT}

A concept and specification of the Accelerator Exchange Format has been introduced in 1998 as a result of the US-LHC collaboration effort aiming to facilitate a lattice exchange among various accelerator programs. The initial version, Standard eXchange Format (SXF[8]), offered a flat operational view of the accelerator state. It was designed after merging two concepts: the MAD sequence of accelerator elements and the UAL normalized sets of element attributes. Since that time, SXF has been employed in the RHIC offline modeling infrastructure and has served as an interface in the MADX-UAL suite comprising design and simulation algorithms. On the other hand, changes in computer technologies and accelerator applications have gradually accumulated a set of new requirements which have eventually resulted into the next enhanced version. The development of the new specification, ADXF 2.0, has to some extend evolved out of E. Forest's idea of "fiber bundles"[9] or the element frame concept dealing with positioning of installed elements. The segregation of position coordinates from element attributes addresses several application issues, such as sharing of common elements by several sectors, supporting of design and operational accelerator descriptions, connecting of accelerator and detector models, and others. Figure 3 shows the structure of the ADXF object model and the relationship of the element frame with other accelerator components.

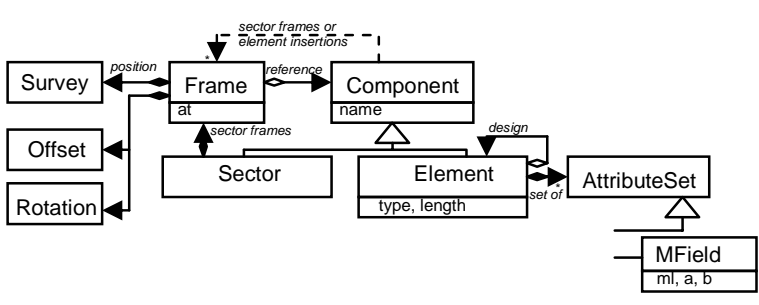

Figure 3: ADXF 2.0 model.

The model is built from five main concepts:

- An accelerator is any sector selected by user;

- A sector is a named sequence of frames with installed accelerator components;

- A frame is a layout of an installed component. It contains a relative position, misalignments, and a reference to an associated sector or accelerator element;

- An accelerator element is an accelerator device or positioned physical effect, such as beam-beam effect. All accelerator elements have the same structure: name, length, and an open collection of attribute sets. An element may also have a reference to its design version.

- An element attribute set is a container of attributes relevant to the single physical effect or feature, such as magnetic field, aperture, etc. 
The same conceptual model has been directly mapped into the XML schema and applied to the definition of structure and syntax of the ADXF file. The choice of the XML language has been determined not only by a rich set of supporting tools but also its extensibility. In ADXF 2.0, the XML inheritance and substitution mechanisms allow one to extend a list of element types and introduce new sets of attributes. For example, all MAD elements are implemented as descendents of the ADXF generic accelerator component, and we can use the conventional MAD terminology with the XML flavor:

<elements>

$<$ marker name="mk1" />

<sbend name="bend" l="lq" angle="deltheta" />

<quadrupole name="quadhv" l="lq" k1="kq1" />

$</$ elements>

As we mentioned earlier, the MAD-oriented design description can be connected or mixed with another view based on a generic element and a collection of attribute sets, for example:

<elements>

$<$ sbend name="d0mp08" l="3.58896" angle="-0.0151186" />

<element name="bi8-dh0" design=" d0mp08">

$<$ mfield b="0 00.0054760 .033503 "

$$
\mathrm{a}=" 0.0-0.0101660 .024366 " />
$$

$</$ element $>$

$\cdots$

$</$ elements $>$

where the <bend> and <mfield> tags are correspondingly associated with sets of bend and magnetic field attributes. The generic (flat) approach is designed after SXF and used for the operational description of the accelerator state.

\section{STATUS AND FUTURE DIRECTIONS}

The interface between the online and off-line models has been started with two "fixed" points: the online OptiCalc Writer and off-line UAL Reader of the ADXF file.

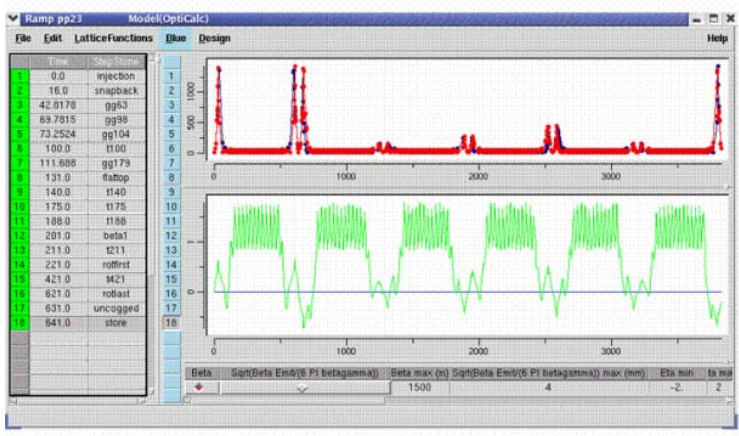

Figure 4: Twiss functions in the online Ramp Editor.
The lattice data generated on the online side has been processed with the UAL parser and successfully crosscompared in both environments. For example, Figure 4 and 5 illustrate the calculated Twiss functions displayed correspondingly in the online Ramp Editor and off-line Interactive Player.

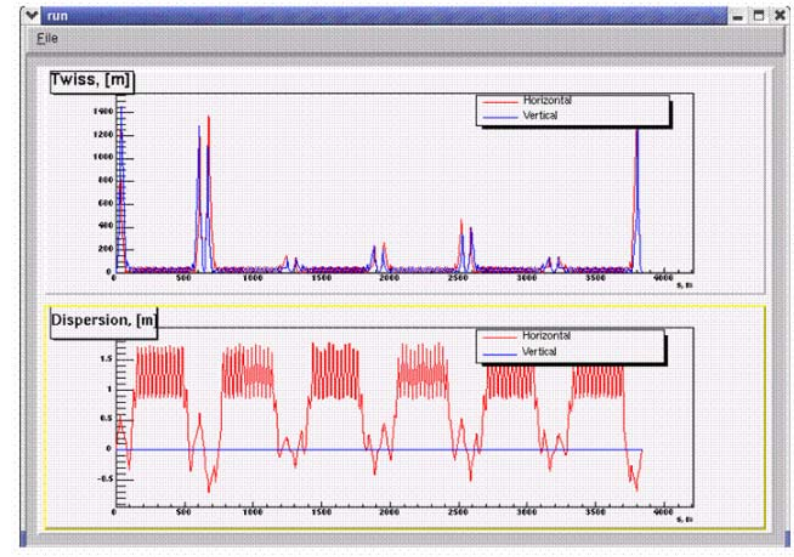

Figure 5: Twiss functions in the off-line UAL Interactive Player.

The scope of practical applications is quite broad. First, the operational lattice data is important for accurate offline beam dynamics studies including non-linear errors, beam-beam compensation, intra-beam scattering, electron cooling, and others. Second, we plan to reuse the off-line environment for development and testing online extensions. Currently, OptiCalc contains only of linear optics. But have recent advances in the orbit and coupling measurements set up a basis for extending the model with new algorithms. Finally, we consider this effort as a yearly step towards integrating AGS and RHIC modeling software.

\section{REFERENCES}

[1] N.Malitsky and R.Talman, "Accelerator Description Exchange Format”, ICAP 1998.

[2] J. van Zeijts, "CDEV Generic Servers for RHIC Commissioning and Operations", ICALEPCS 1999.

[3] T.Satogata et al. "The RHIC/AGS Online Model Environment: Design and Overview”, PAC 1999.

[4] J. Kewisch, J. van Zeijts, S.Peggs, T.Satogata, "Ramp Management in RHIC", PAC 1999.

[5] http://www.ual.bnl.gov

[6] N.Malitsky and R.Talman, "Framework of Unified Accelerator Libraries", ICAP 1998.

[7] http://root.cern.ch

[8] H. Grote, J.Holt, N.Malitsky, F.Pilat, R.Talman, C.G.Trahern, "SXF (Standard eXchange Format): definition, syntax, examples. RHIC/AP/155 (1998)

[9] E. Forest et al. "Polymorphic Tracking Code PTC", KEK Report 2002-3 